NiLs Bulling WIEBE VAN DER HOEK

\section{Special Issue on Logical Aspects of Multi-Agent Systems}

\title{
Preface
}

There is a growing interdisciplinary community of researchers and research groups working on logical aspects of MAS from the perspectives of logic, artificial intelligence, computer science, game theory, etc. The workshop Logical Aspects of Multi-Agent Systems (LAMAS) serves the community as a platform for presentation, exchange, and publication of ideas. The idea for and the name of LAMAS actually emerged independently at two locations: in 2002 and 2007, Hans van Ditmarsch organised two editions of LAMAS in Dunedin, New Zealand. From 2010, the community around the International Conference on Autonomous Agents and Multi-Agent Systems $(A A M A S)$ saw the need for a workshop on logical aspects of multi-agent systems, and the workshop LAMAS has since been held in Toronto, Canada (2010), Osuna, Spain (2011), Valencia, Spain (2012), Toulouse, France (2013), and Paris, France (2014), with the editions in even years being colocated with AAMAS.

In this special issues we present the post-proceedings of the 7th LAMAS, together with extended versions of selected papers of the 15th AAMAS, which were both held in Paris (2014). We invited authors of selected papers to submit extended versions of their papers to this special issue. All submissions had to pass a fresh selection process according to the standards of Studia Logica. We believe that the two selection processes resulted in a collection of papers of high quality. The contributions address different facets of logical aspects of multi-agent systems, including the decidability of multi-agent logics, model checking, logics for agent/robot reasoning and game-theoretical aspects. 
In the first contribution, The Undecidability of Quantified Announcements, Ågotnes, van Ditmarsch and French study the (un-)decidability of three public announcements logics. The presented results are mainly negative: reasoning about announcements is undecidable for two or more agents. This holds for public announcements of arbitrary formulae in the sense of "there is some announcement such that..." (APAL), for group announcements (GAL), as well as for announcements in an adversarial setting (CAL) where agents outside the announcing coalition make announcements themselves. Key contributions of the paper are novel reductions of a tiling problem that establish the undecidability results already in the presence of just two agents. The single agent fragments remain decidable.

The next two contributions investigate modal logics to reason about knowledge/beliefs of agents and their associated model checking problems. Woźna-Szcześniak and Zbrzezny consider in Checking EMTLK Properties of Timed Interpreted Systems via Bounded Model Checking the model checking problem with respect to real-time properties in multi-agent systems with incomplete information. The authors put forward an epistemic extension of Metric Temporal Logic and a SAT-based bounded model checking technique for the existential fragment of their logic over timed models. Chen, Primiero, Raimondi and Rungta propose A Computationally Grounded, Weighted Doxastic Logic, extending Computation Tree Logic, to reason about knowledge and weighted beliefs. Two semantics are proposed to measure the degree of beliefs. One semantics is based on the number of satisfying states consistent with the agent's current indistinguishability class; the other one is of probabilistic nature. It is shown that the model checking problem with respect to both logics can be solved in time polynomial in the size of the model and the formula.

The previous work considers the verification of multi-agent systems from an external point of view. Two further contributions focus upon the agent perspective. The decision making of agents with incomplete information about the world is investigated in Progression and Verification of Situation Calculus Agents with Bounded Beliefs by De Giacomo, Lespérance, Patrizi and Vassos in the setting of the Situation Calculus. The authors show how to progress an agent's belief state and prove that the verification of online executions is decidable in the presence of bounded action theories. Belle and Levesque base their work presented in A Logical Theory of Localization also on the Situation Calculus. They show how action theories capable of expressing probabilistic beliefs in a continuous setting can be used by mobile robots with (possibly) noisy sensors to reason about their spatial locations. 
Finally, social influence and the outcome of rational interactions are subject of the two final papers in the present compilation. The success of an agent to satisfy its goal often depends on the actions of other agents. Thus, it is only natural that agents' decisions influence each other, and it comes as no surprise that the ability of taking good strategic decisions is of key importance in multi-agent interactions. In the paper A STIT Logic for Reasoning About Social Influence, Lorini and Sartor propose a formal framework based on a variant of STIT logic ("seeing to it that") that allows to study and to reason about social influence among rational agents. In the contribution Hard and Soft Preparation Sets in Boolean Games, Harrenstein, Turrini and Wooldridge characterise rational outcomes of Boolean games (which are compact computational models of strategic interactions) and investigate methods to modify them. Agents' rational behavior is captured by preparation sets, which generalize the well-known Nash equilibrium solution concept. The authors investigate techniques, based on side-payments and coalition merging, with respect to their suitability to eliminate undesirable rational outcomes.

We would like to thank all authors contributing to this special issue, the reviewers and the members of the programme committee of LAMAS. Last but not least, we thank Jacek Malinowski for the patience and support that made this volume possible.

Open Access. This article is distributed under the terms of the Creative Commons Attribution 4.0 International License (http://creativecommons.org/licenses/by/4.0/), which permits unrestricted use, distribution, and reproduction in any medium, provided you give appropriate credit to the original author(s) and the source, provide a link to the Creative Commons license, and indicate if changes were made.

\author{
N. Bulling \\ Department of Intelligent Systems \\ Delft University of Technology \\ Delft, The Netherlands \\ n.bulling@tudelft.nl \\ W. VAN DER HOEK \\ Department of Computer Science \\ University of Liverpool \\ Liverpool, UK \\ wiebe@liverpool.ac.uk
}

